\title{
Detección de efectos secundarios asociados a la administración de tramadol y dipirona en un hospital de alta complejidad
}

\author{
Giovanny Alberto Montoya, Claudia Vaca, María Fernanda Parra \\ Departamento de Farmacia, Hospital Universitario de La Samaritana, Universidad Nacional de Colombia, \\ Bogotá, D.C., Colombia
}

Introducción. Un objetivo prioritario después de la comercialización de medicamentos, como la dipirona y el tramadol, en grupos particulares de población, como el colombiano, es garantizar la eficacia y la seguridad.

Objetivo. Describir y estimar la frecuencia de efectos secundarios, incluida la falla terapéutica, asociados al uso de tramadol y a la dipirona en el Hospital Universitario de La Samaritana (III nivel).

Materiales y métodos. Se hizo seguimiento intensivo de los efectos secundarios asociados a la dipirona y al tramadol en los pacientes hospitalizados en los Servicios de Medicina Interna, Ortopedia y Cirugía, durante un período de seis meses como parte de un proyecto a un año. La información se recolectó mediante el formato para reporte al Instituto Nacional de Vigilancia Médica y Alimentos, INVIMA. La probabilidad de causalidad se estableció mediante el algoritmo de la Organización Mundial de la Salud. Se calcularon los costos directos desde la perspectiva del pagador.

Resultados. Se detectaron 213 efectos secundarios en 171 de los 2.547 pacientes que ingresaron a los servicios (proporción de incidencia, 8,4\%). El 53,4\% se clasificó como posible para la dipirona y $46,82 \%$ para el tramadol; el $0,62 \%$, (16 casos) correspondió a efectos secundarios serios. El sistema más frecuentemente afectado fue el gastrointestinal: dipirona, 27\%, y tramadol, 42,9\%. El costo generado por la atención de los efectos secundarios a los medicamentos del estudio fue de US\$14.346,53.

Conclusiones. El impacto negativo de los efectos secundarios de los medicamentos, los recursos que se emplean en su atención y la capacidad de prevenirlos, demuestran que se requieren programas institucionales de farmacovigilancia.

Palabras clave: dipirona/efectos secundarios, tramadol/efectos secundarios, costos en medicamentos, farmacoepidemiología.

\section{Adverse events associated with tramadol and dipirona administration in a level III hospital}

Introduction. The efficacy and safety of pharmaceutical drugs such as dipirone and tramadol must be a primary objective in the post-marketing period and as they are used in specific population groups.

Objectives. The frequency of adverse effects (including therapeutic failure) with the medications tramadol and dipirona were described and estimated.

Material and methods. At the Hospital Universitario de la Samaritana, Bogotá, D.C., Colombia, adverse events associated with dipirone and tramadol were rigorously tracked in patients hospitalized in the internal medicine, as well as the orthopedics and surgery departments. For a period of six months, data were collected by means of the Instituto Nacional de Vigilancia Médica y Alimentos (INVIMA) standard report form. Direct costs of adverse event treatment to the patient were calculated.

Results. Adverse reactions were detected 213 times in 171 (8.4\%) of the 2,547 patients admitted to the services (incidence rate. Of these instances, $53.4 \%$ were rated as possible for dipirone and $46.82 \%$ for tramadol. Of the total, $0.6 \%$ (16 cases) were classes as serious adverse events. The gastrointestinal system was the most affected, with the incidences of adverse events for dipirone 
of $27 \%$ ) and tramadol of $42.9 \%$. The total cost generated by the medical response to the 213 adverse events was estimated to be US\$14,346.53.

Conclusions. An unacceptable level of preventable adverse events was described that impacted the well-being of patients, as well as the costs associated with remedial treatment. These data recommend that institutional pharmacovigilance programs be required.

Key words: Dipyrone/adverse effects, tramadol/adverse effects, drug costs, pharmacoepidemiology.

La dipirona, también denominada metamizol, es un analgésico no narcótico de la familia de las pirazolonas, con actividad antiinflamatoria moderada y buena actividad analgésica y antipirética, pero con la potencialidad, como otros medicamentos, de producir efectos secundarios graves. Suecia y otros países industrializados, como Estados Unidos, retiraron el uso de dipirona hacia la década de los setenta, debido a los casos de agranulocitosis y anemia aplásica asociados a su uso, cuya mortalidad está cercana a $10 \%$, y que se asocia a otras manifestaciones clínicas más comunes de tipo infeccioso, tales como amigdalitis, faringitis, estomatitis o neumonía (1). La incidencia de discrasias sanguíneas con la dipirona fue estimada en uno de cada 3.000 usuarios y, en 1981, The Deutsche Bundesgesundheitsamt calculó el riesgo de agranulocitosis con dipirona en uno de cada 20.000 usuarios por año, razón por lo cual también restringió su uso y prohibió todas sus indicaciones (2). No obstante, en Colombia y otros países, entre los que se incluyen Alemania, Italia, Francia, España, Suiza, Holanda y toda América Latina desde 1943, y la mayoría de los países asiáticos y africanos, continúan la comercialización de este fármaco.

"Las cifras varían en el tiempo, pero se calcula que al cabo de un año, se producen unos 220 millones de tratamientos con dipirona en América Latina. Es decir, que cada día se inician unos 600.000 tratamientos con este medicamento. Específicamente en Colombia, unos ocho millones de personas utilizan cada año medicamentos que contienen dipirona" (3).

Correspondencia:

Giovanny Montoya, Carrera 20 № 187-71, casa 43, Bogotá, D.C., Colombia

Teléfono: (571) 6734520

gamontoyapi@unal.edu.co

Recibido: 02/09/08; aceptado:10/03/09
En 1986, el International Aplastic Anemia and Agranulocytosis Study, Ilamado Estudio Boston, demostró grandes diferencias en la variación de riesgo en diferentes países, que podría explicarse por diferentes factores, como consumo y frecuencia del uso de dipirona, susceptibilidad genética de la población a la agranulocitosis y otros factores propios, y reportó una incidencia de agranulocitosis inducida de uno por cada 1'100.000 usuarios por semana. La dipirona claramente causa agranulocitosis, pero existe información insuficiente para cuantificar adecuadamente el riesgo (4). Es por esto que el determinar el balance riesgo/beneficio y los costos que se generan por los efectos secundarios asociados al uso de dipirona, es importante para los países en los que aún se comercializa, incluyendo Colombia.

Existen varias proyecciones, estudios observacionales y retrospectivos a partir de consumo del medicamento, que muestran que la incidencia de agranulocitosis asociada con la dipirona en América Latina es realmente baja y significativamente menor que la reportada en países como Suecia. Por ejemplo, el estudio de Sollero et al. realizado en Brasil, abarcó un período de 20 años, de 1954 a 1974, con 531.261 pacientes. Se registraron 15 casos de agranulocitosis por pirazolonas (incidencia $<0,002 \%$ ) y un caso por dipirona (incidencia< $<0,0002 \%$ ) (5). En el "Estudio de prevalencia de agranulocitosis inducida por drogas en São Pãulo, Brasil" de Nelson Hamerschlakl, que se llevó a cabo de septiembre de 1981 a marzo de 1990, en el cual se analizaron 19.385 historias clínicas, se encontraron cinco casos de agranulocitosis y sólo se confirmó en un caso la relación entre dipirona y el efecto secundario, con una incidencia de 0,44 a 0,82 casos por millón de pacientes al año. 
En Argentina, el "Estudio retrospectivo de prevalencia de agranulocitosis en el medio hospitalario argentino" de Daniel Goldenberg et al., en el que se analizaron 1'100.000 conteos de laboratorio en siete hospitales de la República Argentina, se encontraron seis casos en tres años de evaluación retrospectiva. En México, Jorge Bernardo Vargas Correa et al. elaboraron el estudio "Metamizol. Evaluación del riesgo para agranulocitosis y anemia aplásica", en el que se revisaron los registros del Servicio de Hematología del Hospital Regional Mérida del Instituto de Seguridad y Servicios Sociales de los Trabajadores del Estado (ISSSTE) desde 1992, y también se evaluaron los registros de los 10 años previos del Servicio de Medicina Interna (ingresos por agranulocitosis o anemia aplásica), así como los de consumo de medicamentos y metamizol del hospital mediante los registros de la farmacia de tres años a la fecha; se encontraron tres casos de agranulocitosis y cuatro de anemia aplásica en tres años de investigación retrospectiva; ninguno de ellos se asoció al uso de metamizol. La incidencia de anemia aplásica reportada en estudios mexicanos fue mayor, con 3,9 por millón de individuos al año (6).

El tramadol es un analgésico narcótico, derivado sintético de la codeína, utilizado para el control del dolor moderado a grave, como la mayoría de los opiáceos.

Cossmann et al. (1997) consideraron la información proveniente de los estudios clínicos de fase II a IV y después de la comercialización, y recolectaron datos provenientes de cerca de 21.000 pacientes, y encontraron que los efectos secundarios más frecuentes fueron: náuseas $(6,1 \%)$, desvanecimiento $(4,6 \%)$, somnolencia $(2,4 \%)$, cansancio/fatiga $(2,3 \%)$, sudoración $(1,9 \%)$, vómito $(1,7 \%)$, boca seca $(1,6 \%)$, y alguna reacción de hipersensibilidad dada por alergias o reacciones anafilácticas $(0,1 \%)(7)$.

En el estudio publicado por Tribiño y Maldonado, realizado en el Hospital Universitario de La Samaritana, se evaluaron los costos directos y los aspectos clínicos de las efectos secundarios a medicamentos en pacientes hospitalizados en el servicio de medicina interna; arrojó resultados que incluyeron la detección de "268 reacciones adversas en 208 de los 836 pacientes que ingresaron al servicio (proporción de incidencia de $25,1 \%$ y razón de presencia de $0,32)$. El $74,3 \%$ de los EAM se clasificó como probable, dentro de (sic.) las cuales el $92,5 \%$ fue tipo A. El 81,3\% correspondió a reacciones moderadas, encontrándose que el sistema más frecuentemente afectado fue el hematológico $33,9 \%$ y que los medicamentos que actúan en sangre fueron los más frecuentemente relacionados $37,6 \% "(8)$.

Dada la frecuencia del uso en el Hospital Universitario de La Samaritana, el perfil de seguridad y el comportamiento creciente del reporte de efectos secundarios, tanto en el ámbito institucional como en el nacional (Vacca C, Ocampo M. Avances del programa de farmacovigilancia en el HUS. Póster presentado en el III Encuentro Internacional de Farmacovigilancia; septiembre de 2006; Bogotá, D.C.) (9), para los fármacos descritos, se presentó la propuesta del estudio de seguimiento activo de los efectos secundarios asociados a los medicamentos tramadol y dipirona con un análisis de los costos directos desde la perspectiva del pagador, con el fin de establecer incidencias de efectos secundarios, detección de señales de seguridad y plantear estrategias de prevención y manejo en pro de los pacientes y de un uso adecuado de los medicamentos (10), mediante la búsqueda activa en dos fases de investigación de seis meses cada una y la recolección de datos de un año.

\section{Materiales y métodos}

Se realizó una búsqueda activa combinada con reporte voluntario de casos de los efectos secundarios asociados a los medicamentos dipirona y tramadol en sus presentaciones inyectables. Para este fin, se revisaron diariamente todas las historias clínicas de los pacientes a los que fueron prescritos dichos fármacos con seguimiento médico de cada caso, durante el período comprendido entre el $1^{\circ}$ de marzo de 2007 y el 15 de septiembre de 2007; se trató de un estudio descriptivo, observacional con seguimiento de casos. 
Se incluyeron pacientes sin restricciones de edad o sexo que fueron hospitalizados en los servicios de medicina interna, ortopedia y cirugía general del Hospital Universitario de La Samaritana, por ser los de mayor volumen de prescripción de dipirona y tramadol; también se incluyeron aquellos pacientes que ingresaron por sospecha de efectos secundarios relacionados con estos dos medicamentos.

Para el diseño del estudio no se tuvo en cuenta ningún criterio de exclusión. No se realizó un cálculo de muestra, ya que se incluyó la totalidad de los pacientes del hospital a quienes se prescribieron dichos medicamentos. El periodo elegido (1을 de marzo a 15 de septiembre de 2007) corresponde al primer periodo de recolección de datos de seis meses de la primera fase del estudio, debido a que se programó una segunda fase de toma de datos por seis meses más, para completar un año de farmacovigilancia intensiva dirigida a los fármacos ya descritos, de acuerdo con el diseño planteado inicialmente.

La identificación de los pacientes con prescripción de los fármacos en estudio, se realizó mediante la consulta prediseñada por los departamentos de Informática y Farmacia del hospital. Una vez identificados los pacientes, la información se recolectó en el Formato de Reacciones Adversas a Medicamentos (FORAM) del Instituto Nacional de Vigilancia Médica y Alimentos (INVIMA) y se digitó en una hoja electrónica de Microsoft Excel® para su procesamiento.

Para las variables cuantitativas continuas, se calcularon el promedio y la desviación estándar, y para las variables cualitativas, se estableció la proporción expresada como porcentaje. La relación causal se estableció mediante el algoritmo y las categorías de causalidad de la Organización Mundial de la Salud (OMS). El registro de los efectos secundarios se realizó utilizando el diccionario WHOART (World Health Organization Adverse Reaction Terminology). El equipo de investigación reportó las sospechas de efectos secundarios a los medicamentos.

\section{Análisis de datos}

El análisis de datos se realizó con base en los resultados obtenidos en la primera fase del estudio, que corresponde a seis meses de recolección activa.

Los datos recopilados se analizaron para:

describir y estimar la frecuencia de efectos secundarios a medicamentos, entendidos como cualquier situación médica desfavorable experimentada por un paciente en tratamiento con dipirona y tramadol, sin que necesariamente exista una relación de causalidad con el medicamento e incluyendo la falla terapéutica (falta de respuesta o beneficio clínico para el cual se administró el medicamento), asociados a su uso en el Hospital Universitario de La Samaritana (III nivel) en un periodo de un año;

caracterizar sociodemográficamente la población que presentó efectos secundarios asociados al uso de tramadol y dipirona, identificar las indicaciones para las cuales fueron prescritos, así como las alteraciones asociadas a la utilización de los mismos;

identificar los esquemas terapéuticos asociados a los fármacos sometidos a vigilancia activa, clasificar la causalidad y seriedad de los efectos secundarios detectados, generar alertas sobre los efectos secundarios serios a la dipirona y al tramadol, con el fin de proveer información para la orientación de decisiones y posibles estrategias de prevención, y realizar una estimación de los costos directos de los efectos secundarios (desde la perspectiva del pagador) detectados como prevenibles con los medicamentos del estudio.

El tipo de efectos secundarios en los que se determinó como definitiva su relación de causalidad con el algoritmo de la OMS, se distribuyeron según la clasificación de Rawlins y Thopson en: A: incrementada (augmented); B: rara (bizarre), y E: terminal (end) (11).

El diagnóstico principal de los casos se definió según la CIE-10. El criterio de seriedad se clasificó de acuerdo con las guías ICH-E2A, como cualquier situación médica desfavorable en la que el fármaco a cualquier dosis, causara la muerte, hubiese puesto en riesgo la vida del paciente, incapacitara 0 inhabilitara permanentemente, hubiese requerido o prolongado la 
hospitalización del paciente, hubiese conducido a anomalías congénitas y cualquier efecto médicamente significativo.

\section{Aspectos éticos}

Teniendo en cuenta las disposiciones consignadas en las "Normas científicas, técnicas y administrativas para la investigación en salud", según la Resolución № 8430 de 1993, este proyecto se clasificó en la categoría de investigación sin riesgo, puesto que "los métodos y estrategias propuestas no involucran intervención o modificación intencionada de variables biológicas, fisiológicas o sociales de los individuos bajo estudio o la identificación de aspectos sensitivos de su conducta" (Título II, Capítulo I, Artículo 11), por lo cual se obvió la obtención del consentimiento informado.

El presente estudio fue aprobado por el Comité de Investigaciones de la Facultad de Ciencias de la Universidad Nacional de Colombia y el Comité de Investigaciones del Hospital Universitario de La Samaritana.

\section{Resultados}

Durante el período de vigilancia activa, se realizó seguimiento a las historias clínicas de 2.547 pacientes a quienes se prescribieron los medicamentos del estudio en los servicios de medicina interna, ortopedia y cirugía general.

Con un promedio de edad de 58,9 $\pm 0,67$ años, la media de los pacientes con efectos secundarios a la dipirona fue de $46,4 \pm 21,8$ años y de $51,5 \pm 18,8$ años para el tramadol, con $25,35 \%$ de los pacientes con edades mayores de 65 años.

Se detectaron 213 efectos secundarios a los medicamentos del estudio, en 171 pacientes de los 2.547 evaluados, con una proporción de incidencia (cociente entre número de pacientes con efectos secundarios y total de pacientes ingresados) de $8,4 \%$. Los efectos secundarios a los medicamentos del estudio se presentaron en 95 hombres $(55,5 \%)$ y 76 mujeres $(44,4 \%)$.

$A$ los pacientes que presentaron efectos secundarios por la dipirona, se les administró en promedio $7 \pm 2$ medicamentos (rango, 3 a 13), y se encontraron $21 / 87$ pacientes, es decir,
$24,1 \%$, con tratamientos que incluían ocho o más medicamentos; por otra parte, a los pacientes que presentaron efectos secundarios al tramadol se les administró en promedio $7 \pm 3$ medicamentos (rango, 2 a 14), y se encontraron $39 / 84$ pacientes, es decir, $46,4 \%$, con tratamientos que incluían ocho o más medicamentos. La estancia hospitalaria para pacientes con efectos secundarios a la dipirona fue de $12,44 \pm 9,07$ días y de $9,28 \pm 7,32$ para el tramadol, y el promedio de días de estancia hospitalaria adicional para pacientes con efectos secundarios a la dipirona fue, en promedio, de 1,95 días con un rango entre 1 a 5 días y de 2,25 días con un rango entre 1 a 7 días para el tramadol.

La dipirona se prescribió principalmente como analgésico (96\%) y sólo en $4 \%$ de los casos como antipirético, con diferentes esquemas terapéuticos resumidos en el cuadro 1.

Cabe resaltar que la dosis máxima recomendada para la dipirona es de $4 \mathrm{~g}$ al día, por vía intramuscular o intravenosa, por los periodos más cortos posibles (no más de una semana consecutiva) (12). Por otra parte, el tramadol se prescribió como analgésico en el $100 \%$ de los pacientes, utilizando los esquemas terapéuticos resumidos en el cuadro 2. De igual forma, en la información básica de prescripción para el medicamento se aclara que la dosis máxima recomendada de tramadol para pacientes adultos, mayores de 14 años, es de hasta 400 mg al día (12).

Los efectos secundarios a la dipirona y al tramadol comprendieron 51 manifestaciones diferentes,

Cuadro 1. Esquemas terapéuticos utilizados en los pacientes que presentaron efectos secundarios a quienes se prescribió dipirona.

\begin{tabular}{lrr}
\hline $\begin{array}{l}\text { Esquema terapéutico } \\
\text { utilizado }\end{array}$ & Frecuencia & Porcentaje \\
\hline 5 g cada 8 horas, IV & 1 & 1,35 \\
2,5 g cada 6 horas, IV & 51 & 68,90 \\
2,5 g cada 8 horas, IV & 16 & 21,62 \\
2,0 g cada 6 horas, IV & 1 & 1,35 \\
1,0 g cada 8 horas, IV & 1 & 1,35 \\
1,0 g cada 6 horas, IV & 4 & 5,40 \\
\hline
\end{tabular}

IV: intravenoso 
de las cuales, la más frecuente para la dipirona fue la falla terapéutica $(21,59 \%)$, entendida como la ausencia del efecto terapéutico deseado por el médico tratante; las demás se presentan en el cuadro 3. Durante la observación hecha en este estudio, no se encontraron reportes de agranulocitosis asociados a la dipirona y los casos de compromiso hematológico, que alcanzaron una incidencia de 15,9\%, correspondieron a seis casos de leucopenia $(83,3 \%$ evaluados como posible) y cinco de anemia ( $80 \%$ evaluados como improbable), de acuerdo con el algoritmo de causalidad de la OMS.

No se encontró ningún caso de agranulocitosis con dipirona en este estudio, lo cual puede explicarse por varios factores, entre los que se incluyen un diseño experimental que no permite la detección de casos de este tipo, el uso distinto de dipirona entre países o la susceptibilidad

Cuadro 2. Esquemas terapéuticos utilizados en los pacientes que presentaron efectos secundarios a quienes se prescribió tramadol.

\begin{tabular}{lcc}
\hline $\begin{array}{l}\text { Esquema terapéutico } \\
\text { utilizado }\end{array}$ & Frecuencia & Porcentaje \\
\hline 17 mg por hora, infusión & 2 & 2,06 \\
100 mg cada 8 horas, IV & 7 & 7,21 \\
50 mg cada 6 horas, IV & 2 & 2,06 \\
50 mg cada 8 horas, IV & 73 & 75,20 \\
50 mg cada 12 horas, IV & 8 & 8,24 \\
25 mg cada 6 horas, IV & 2 & 2,06 \\
25 mg cada 8 horas, IV & 4 & 4,12 \\
\hline
\end{tabular}

IV: intravenoso genética de ciertos grupos de población a desarrollar agranulocitosis.

Para el tramadol, el estreñimiento fue el efecto secundario más frecuente $(23,02 \%)$ y hubo dos casos de íleo adinámico que correspondieron a $1,59 \%$ de los efectos encontrados, con una incidencia de 0,037 por 100 pacientes al año (cuadro 4).

Al aplicar el algoritmo de la OMS para determinar la probabilidad de causalidad, la proporción de efectos secundarios a dipirona se clasificó como: posible $(53,4 \%)$, improbable $(30,68 \%)$, definitiva $(9,09 \%)$, probable $(3,4 \%)$ y no clasificada $(3,4 \%)$. En cuanto al tramadol, la proporción de efectos secundarios se clasificó como: posible $(46,82 \%)$, definitiva $(30,15 \%)$, probable $(12,69 \%)$ e improbable (10,31\%).

De acuerdo con la clasificación de seriedad, la mayoría de los efectos secundarios a dipirona se clasificaron como no serios $(93,18 \%)$ y los otros como serios $(6,81 \%)$, mientras que, para el tramadol, fueron de $92,06 \%$ y $7,93 \%$, respectivamente.

El principal sistema comprometido por los efectos secundarios a la dipirona y al tramadol fue el gastrointestinal, principalmente, náuseas, vómito y estreñimiento (cuadros 5 y 6 ). Las principales 10 categorías diagnósticas de los pacientes con efectos secundarios a la dipirona y al tramadol, se presentan en los cuadros 7 y 8 .

Cuadro 3. Efectos secundarios más frecuentes en los pacientes a quienes se prescribió dipirona.

\begin{tabular}{lcccc}
\hline $\begin{array}{l}\text { Efectos secundarios } \\
\text { a dipirona }\end{array}$ & Frecuencia & Porcentaje & $\begin{array}{c}\text { Incidencia por 100 } \\
\text { pacientes/año }\end{array}$ & $\begin{array}{c}\text { Porcentaje global } \\
\text { reportado (15) }\end{array}$ \\
\hline Falla terapéutica & 19 & 21,59 & 0,029 & ND \\
Vómito/náuseas & 13 & 14,77 & 0,016 & $16(25)$ \\
Erupción cutánea & 7 & 7,95 & 0,025 & $<0,01->0,001$ \\
Prurito generalizado & 6 & 6,82 & 0,0075 & $<0,01->0,001$ \\
Leucopenia & 6 & 6,82 & 0,0075 & $<0,01->0,001$ \\
Anemia & 5 & 5,68 & 0,0064 & $\mathrm{ND}$ \\
Taquicardia & 5 & 5,68 & 0,0064 & $\mathrm{ND}$ \\
Disnea & 4 & 4,55 & 0,0051 & $<1$ \\
Estreñimiento & 2 & 2,27 & 0,0025 & $<0,01$ \\
Trombocitopenia & 2 & 2,27 & 0,0025 & \\
Total, 10 primeras causas & & 78,41 & & \\
\hline
\end{tabular}


De los efectos secundarios a la dipirona, 43 $(48,86 \%)$ generaron costos por diferentes conceptos, discriminados de la siguiente forma: a) 28 de $88(31,8 \%)$ requirieron medicamentos como parte del tratamiento de los efectos secundarios; el costo por concepto de medicamentos utilizados por esta causa fue de US\$1.398,59; b) 16 de $88(18,18 \%)$ requirieron realización de exámenes paraclínicos adicionales, el costo por este concepto fue de US\$352,54 (tarifa SOAT); c) 6 del total de efectos secundarios detectados $(6,8 \%)$ requirieron la realización de diferentes procedimientos cuyo costo fue de US $\$ 193,43$ (tarifa SOAT); d) en 10 de los efectos secundarios $(11,36 \%)$ se solicitaron consultas a otros servicios, cuyo costo fue de US\$151,68 (tarifa SOAT); ninguno de los efectos secundarios detectados requirió traslado del paciente a la unidad de cuidados intensivos o intermedio, y e) el costo por estancia hospitalaria adicional fue de US\$ 6.639,48 (tarifa SOAT).

Por otra parte, de los efectos secundarios al tramadol, 55 (43,65\%) generaron igualmente costos por diferentes conceptos, discriminados de la siguiente forma: a) 44 de 126 (34,9\%) requirieron medicamentos como parte del tratamiento de los efectos secundarios; el costo por este concepto fue de US\$ 313,27 ; b) 6 de $126(4,76 \%)$ requirieron realización de exámenes paraclínicos adicionales; el costo por este concepto fue de US\$ 118,74 (tarifa SOAT); c) 4 del total de efectos secundarios a medicamentos detectados $(3,17 \%)$ requirieron la realización de diferentes procedimientos cuyo costo fue de US\$ 42,02 (tarifa SOAT); d) en 13 de los efectos secundarios (10,31\%) se solicitaron consultas a otros servicios, cuyo costo fue de US\$197,61 (tarifa SOAT).

Cuadro 4. Efectos secundarios más frecuentes en los pacientes a quienes se prescribió tramadol.

\begin{tabular}{lcccc}
\hline $\begin{array}{l}\text { Efectos secundarios } \\
\text { a tramadol }\end{array}$ & Frecuencia & Porcentaje & $\begin{array}{c}\text { Incidencia por 100 } \\
\text { pacientes/año }\end{array}$ & $\begin{array}{c}\text { Porcentaje global } \\
\text { reportado (12) }\end{array}$ \\
\hline Estreñimiento & 29 & 23,02 & 0,054 & $<1$ \\
Vómito/náuseas & 19 & 15,08 & 0,035 & 6,1 \\
Falla terapéutica & 12 & 9,52 & 0,022 & ND \\
Somnolencia & 10 & 7,94 & 0,018 & 2,4 \\
Taquicardia & 7 & 5,56 & 0,013 & 4,6 \\
Vértigo/mareo & 6 & 4,76 & 0,011 & $<1$ \\
Hipotensión & 3 & 2,38 & 0,0056 & $<1$ \\
Epigastralgia & 3 & 2,38 & 0,0056 & $<0,1$ \\
Dificultad respiratoria & 3 & 2,38 & 0,0056 & $<0,1$ \\
Íleo adinámico & 2 & 1,59 & 0,0037 & \\
Total, 10 primeras causas & & 74,61 & & \\
\hline
\end{tabular}

Cuadro 5. Compromisos más frecuentes en los pacientes a quienes se les prescribió dipirona.

\begin{tabular}{lcc}
\hline Compromiso & Frecuencia & Porcentaje \\
\hline Gastrointestinal & 20 & 27,0 \\
Piel & 20 & 22,7 \\
Falla terapéutica & 19 & 21,6 \\
Hematológico & 14 & 15,9 \\
Cardiovascular & 5 & 5,7 \\
Respiratorio & 4 & 4,5 \\
Hidroelectrolítico & 3 & 3,4 \\
Sistema nervioso central y & & \\
sistema nervioso periférico & 3 & 3,4 \\
Total efectos secundarios & 88 & 100,0 \\
\hline
\end{tabular}

Cuadro 6. Compromisos más frecuentes en los pacientes a quienes se les prescribió tramadol.

\begin{tabular}{lcc}
\hline Compromiso & Frecuencia & Porcentaje \\
\hline Gastrointestinal & 54 & 42,9 \\
Sistema nervioso central y & & \\
sistema nervioso periférico & 24 & 19,0 \\
Falla terapéutica & 22 & 17,5 \\
Cardiovascular & 11 & 8,7 \\
Piel & 7 & 5,6 \\
Hematológico & 3 & 2,4 \\
Hidroelectrolítico & 3 & 2,4 \\
Respiratorio & 2 & 1,6 \\
Total efectos secundarios & 126 & 100,0 \\
\hline
\end{tabular}


Ninguno de los efectos secundarios detectados requirió traslado del paciente a la unidad de cuidados intensivos o intermedios, y e) el costo por estancia hospitalaria adicional fue de US\$ 4.939,13 (tarifa SOAT).

Cuadro 7. Categorías diagnósticas de los pacientes que presentaron efectos secundarios a quienes se prescribió dipirona.

\begin{tabular}{lcc}
\hline Categoría diagnóstica & Frecuencia & Porcentaje \\
\hline Politraumatismo con fractura o & & \\
sin ella & 22 & 22,60 \\
Posoperatorio de cirugía de abdomen & 16 & 16,49 \\
Enfermedad arterial oclusiva & 8 & 8,24 \\
Neoplasia de tejidos blandos & 7 & 7,21 \\
Posoperatorio de cirugía de tórax & 5 & 5,15 \\
Enfermedad autoinmune -lupus & & \\
eritematoso sistémico & 4 & 4,12 \\
Neumonía bacteriana no especificada & 4 & 4,12 \\
Insuficiencia renal crónica & 3 & 3,09 \\
Neoplasias de tejidos duros & 3 & 3,09 \\
Trombosis venosa no especificada & 2 & 2,06 \\
Total, 10 primeras causas & & 76,17 \\
\hline
\end{tabular}

Cuadro 8. Categorías diagnósticas de los pacientes que presentaron efectos secundarios a quienes se prescribió tramadol.

\begin{tabular}{lcc}
\hline Categoría diagnóstica & Frecuencia & Porcentaje \\
\hline Fractura simple o compleja & 14 & 18,90 \\
$\begin{array}{l}\text { Posoperatorio de cirugía de } \\
\text { abdomen }\end{array}$ & 11 & 14,86 \\
Politraumatismo con fractura & & \\
o sin ella & 7 & 9,45 \\
Enfermedad arterial oclusiva & 5 & 6,75 \\
Neoplasia de tejidos blandos & 5 & 6,75 \\
Infección de tejidos blandos & 5 & 6,75 \\
Dolor abdominal & 4 & 5,40 \\
Insuficiencia renal crónica & 3 & 4,05 \\
Posoperatorio de cirugía de & & \\
extremidades & 3 & 4,05 \\
Neumonía bacteriana no especificada & 2 & 2,70 \\
Total, 10 primeras causas & & 79,66 \\
\hline
\end{tabular}

En resumen (cuadro 9), el costo total directo por la atención de los efectos secundarios a la dipirona y al tramadol fue de $\$ 26^{\prime} 048,281$ o US\$ 14.347 a una tasa de cambio de Col\$1.815,65 por dólar (13). Para la dipirona, el más costoso fue un caso de leucopenia y, para el tramadol, fue un caso de síndrome de Stevens-Johnson.

Si se acepta un porcentaje de efectos secundarios prevenibles de $70 \%$, el monto ahorrado para el pagador sería de \$18'233.797 o US\$ $10.042,57$.

\section{Discusión}

Desde el punto de vista de distribución por sexo, no se encuentra diferencia de proporciones entre el masculino y el femenino para la presentación de efectos secundarios. Sin embargo, es importante tener en cuenta que estas distribuciones corresponden a datos crudos que deben compararse con los datos de distribución por sexo de la población usuaria del hospital en general, para definir si existe selección particular de los grupos por sexo, lo cual aplica para los datos del promedio global de edad.

Los adultos mayores presentan un riesgo especial de desarrollar efectos secundarios a los medicamentos. La frecuencia de efectos secundarios entre los ancianos es de 2 a 7 veces mayor que en la población menor de 60 años (14); esto es debido en parte al hecho de que los adultos mayores consumen más fármacos, y durante períodos más prolongados, que los más jóvenes $(14,17,18)$. A esto se añade que los adultos mayores son más sensibles a los efectos perjudiciales de ciertos fármacos, como consecuencia de las alteraciones farmacodinámicas y farmacocinéticas, y a la disminución de la capacidad de reserva fisiológica y a la reducción de los mecanismos

Cuadro 9. Distribución de costos de efectos secundarios de acuerdo con su origen (US\$).

\begin{tabular}{lcccrrr}
\hline Origen & $\begin{array}{c}\text { Estancia } \\
\text { hospitalaria }\end{array}$ & Medicamentos & $\begin{array}{c}\text { Exámenes } \\
\text { paraclínicos }\end{array}$ & Interconsultas & Procedimientos & Total \\
\hline Dipirona & $6.639,48$ & 1398,6 & 352,54 & 151,68 & 193,42 & $8.735,73$ \\
Tramadol & $4.939,13$ & 313,28 & 118,74 & 197,61 & 42,02 & $5.610,79$ \\
Total & $11.578,61$ & 1711,88 & 471,28 & 349,29 & 235,44 & $14.346,53$ \\
Porcentaje & 80,71 & 11,93 & 3,29 & 2,43 & 1,64 & 100 \\
\hline
\end{tabular}


homeostáticos corporales. Aunque el conocimiento clínico apoya la existencia de un mayor riesgo de aparición de reacciones adversas asociadas a la edad avanzada, parece que existe una gran variabilidad interindividual en el proceso de envejecimiento -incluyendo la naturaleza no uniforme de los cambios farmacocinéticos y farmacodinámicos en los sujetos de edad avanzada- lo cual indica que la realidad puede ser bastante más compleja. De manera que las características específicas del paciente (fisiológicas y funcionales) son probablemente más importantes que cualquier medida cronológica a la hora de predecir tanto los efectos secundarios, como los beneficios, de cualquier tratamiento farmacológico $(14,19,20)$.

No se encontró ningún caso de agranulocitosis durante la búsqueda en la primera parte de este estudio, lo cual puede explicarse por varios factores, entre los que se incluye un diseño que no permite la detección de casos de este tipo por falta de potencia del estudio para detectarla, dada su baja incidencia, calculada en 1,2 casos de agranulocitosis por un millón de personas expuestas a la dipirona hasta por una semana (4), los hábitos de prescripción diferentes de dipirona entre países, la susceptibilidad genética de ciertos grupos de población a desarrollar agranulocitosis o por otras hipótesis ya planteadas que explican que ni la dipirona ni su metabolito activo 4-metil-amino-antipirina afectan el proceso de diferenciación de los granulocitos ni inducen apoptosis de los granulocitos ya diferenciados, sino que la agranulocitosis tiene un origen inmunoalérgico (21)

La falla terapéutica fue el efecto secundario más frecuente para la dipirona en este estudio, con una incidencia de 0,029 por 100 pacientes al año. Es importante mencionar que a los pacientes a quienes se prescribió como analgésico, fue con el fin de tratar enfermedades que generan dolor de intensidad moderada y grave, y que correspondieron a $60 \%$ de los pacientes medicados (cuadro 7), para cuya seriedad se debe reevaluar el uso de un antinflamatoriono esteroideo, como la dipirona.

Es de destacar que el número de medicamentos concomitantes es un factor fuertemente relacio- nado con el desarrollo de efectos secundarios a los medicamentos (22-24). En este estudio, el promedio de medicamentos administrados a cada paciente fue de siete, con un porcentaje mayor a $24 \%$ en el caso de la dipirona y de $46 \%$ en el caso del tramadol para más de ocho medicamentos por paciente.

Para medicamentos como la dipirona y el tramadol, cuya principal vía de biotransformación es a través del sistema microsómico hepático (citocromo P450), el efecto de las interacciones medicamentosas aumenta significativamente, y el uso de medicamentos concomitantes es un factor esencial sobre la presentación o ausencia de efectos secundarios. Lugian et al. mencionan como factores de riesgo para la presencia de efectos secundarios a medicamentos en los pacientes hospitalizados, la edad y la complejidad de las enfermedades concomitantes, porque el cuidado de estos pacientes requiere mayor intervención (25).

Cabe resaltar que la dosis máxima recomendada para pacientes en quienes se prescribe dipirona es excedida en $94,6 \%$ de los pacientes del Hospital Universitario de La Samaritana, aunque la incidencia de efectos secundarios a la dipirona observados en los servicios de medicina interna, ortopedia y cirugía general no llevaron a hallazgos de seguridad adicionales a los ya descritos para el producto. Sin embargo, se debe hacer especial énfasis en que está siendo adecuadamente utilizado sólo en 5,4\% de las prescripciones evaluadas. De este grupo, los cuatro pacientes que desarrollaron efectos secundarios fueron evaluados como no serios y correspondieron a tres efectos por compromiso dermatológico (erupción cutánea y prurito) y una epigastralgia. Todos los pacientes que presentaron efectos secundarios evaluados como serios tenían prescripción de dipirona a dosis de 2,5 g intravenosos cada 6 horas (dosis diaria total de $10 \mathrm{~g}$ ). Por lo anterior, es importante generar alertas en el Hospital Universitario de La Samaritana con respecto a las sobredosis utilizadas del medicamento, con el fin de crear planes de control y sensibilización del uso adecuado de dipirona. 
En cuanto a las dosis utilizadas para tramadol, se puede concluir que es un medicamento adecuadamente utilizado en los servicios de medicina interna, ortopedia y cirugía general, sin que se encontraran dosis por encima de las recomendadas en los pacientes medicados. Es de esperar que la mayor proporción de efectos secundarios se presente en los pacientes a los que se suministra el medicamento en dosis de $50 \mathrm{mg}$ intravenosos cada 8 horas, dado que es el esquema más ampliamente utilizado en los servicios evaluados.

La mayoría de efectos secundarios se clasificaron como posibles $(53,4 \%$ para la dipirona y $47 \%$ para el tramadol) de acuerdo con el algoritmo de causalidad de la OMS y, como no serios, según el criterio de seriedad; estos hallazgos se obtuvieron de la aplicación del algoritmo a cada caso por dos evaluadores independientes y de acuerdo con el criterio médico de los mismos. Los efectos secundarios definitivos desde el punto de vista de causalidad, se clasificaron como tipo B (100\%), para la dipirona (correspondiente a los casos de falla terapéutica) y como $A$ $(52,6 \%)$ y $(47,3 \%)$ fue de tipo B para el tramadol predominio, lo cual indica que la gran mayoría son predecibles.

El mayor porcentaje de los costos generados fueron causados por el incremento de la estancia hospitalaria de los pacientes que presentaron efectos secundarios a los medicamentos, que en total acumularon 170 días; también, se debe tener en cuenta que mediante este estudio se calcularon los costos directos relacionados directamente con los servicios sanitarios, entre los que se incluyen los costos de medicamentos, el cuidado sanitario, las pruebas diagnósticas, las consultas y la hospitalización. No se tuvieron en cuenta otros como costos por trabajo de personal de enfermería, camilleros y costos directos no sanitarios, entre los que se incluyen los servicios sociales.

Las limitaciones que se presentaron en esta investigación fueron:

es posible que la población del hospital donde se realizó el estudio presente diferencias con respecto a la de otras instituciones, a pesar de que éste es centro de referencia de la red de salud de Cundinamarca, de manera que la asimilación de los resultados a otros hospitales puede ser discutible;

se reconoce que la forma más rigurosa de determinar la estancia hospitalaria adicional es mediante la diferencia entre la estancia del paciente con efectos secundarios a medicamentos y la estancia de un grupo de pacientes control apareados por variables como edad, sexo, diagnóstico y gravedad;

el cálculo de los recursos ahorrados al evitar los efectos secundarios prevenibles usa proporciones publicadas en la literatura; no se utilizó un algoritmo para definir el carácter prevenible de dichos efectos detectados $y$, por ello, no se calculó una proporción de efectos secundarios prevenibles; habría que determinar si las proporciones empleadas se ajustan a las características propias de nuestro medio, y

esta investigación incluyó sólo los servicios de medicina interna, ortopedia y cirugía general, por ser donde se prescribe el mayor porcentaje de los medicamentos del estudio; por lo tanto, dadas las diferentes enfermedades y medicamentos empleados en estos servicios, los efectos pueden no reflejar su comportamiento global en la institución.

Los hallazgos de esta investigación confirman que los efectos secundarios a medicamentos generan un impacto clínico negativo implícito en la evolución de los pacientes, no evaluada objetivamente en este estudio, y aumentan en forma considerable los costos de la atención hospitalaria.

Los programas de farmacovigilancia dirigidos a determinar tendencias 0 señales que permitan modificar, por medio de campañas de prevención y educación, errores de medicación que estén influenciando el sistema negativamente, redundarían en la reducción de la estancia hospitalaria y la morbilidad de los pacientes, y resulta en ahorros considerables para la institución. El mayor ahorro logrado al evitar efectos secundarios a medicamentos, 
prevenibles mediante un programa de esta naturaleza, se daría en términos monetarios y disponibilidad de camas. Además, mejoraría la calidad de la atención y el resultado clínico del paciente. Por otra parte, indudablemente se requiere una mayor integración con el proceso de desarrollo y estatutos dispuestos en los Comités de Farmacia y Terapéutica institucional, teniendo en cuenta la alineación y el cumplimiento del Decreto 2200 de 2005, por el cual se reglamenta el servicio farmacéutico y se dictan otras disposiciones, y la Resolución 1403 de 2007, por la cual se determina el Modelo de Gestión del Servicio Farmacéutico, se adopta el Manual de Condiciones Esenciales y Procedimientos y se dictan otras disposiciones, y por medio del cual se deben fortalecer estos programas de farmacovigilancia.

\section{En conclusión:}

1. El estudio adelantado constituye una iniciativa de investigación conjunta entre la Universidad Nacional de Colombia y el Hospital Universitario de La Samaritana, que ha permitido a la fecha el seguimiento de 27.099 exposiciones a los fármacos en estudio y la detección de 214 efectos secundarios, la mayoría de los cuales se presentaron en pacientes de sexo masculino $(54,3 \%)$ y edades promedio de $49,2 \pm 20,3$ años (media para la dipirona de 46,4 $\pm 21,8$ y de $51,5 \pm 18,8$ para el tramadol).

2. La mayoría de los efectos secundarios ( $\geq 90 \%)$ se clasificaron como no serios y posibles (dipirona, 53,4\%, y tramadol, 46,82\%).

3. El principal sistema comprometido por efectos secundarios a la dipirona y al tramadol fue el gastrointestinal, principalmente, náuseas, vómito y estreñimiento.

4. Aunque del total de efectos secundarios reportados ninguno fue realizado por los médicos tratantes, se destaca la oportunidad de la vigilancia activa en la detección de problemas asociados con el uso, en este caso de la dipirona, la cual fue prescrita como analgésico (96\%) y como antipirético (4\%), en dosis de $2,5 \mathrm{~g}$ intravenosos cada 6 horas en el $68,9 \%$ de los casos, dosis más de tres veces superior a la dosis diaria definida $(3 \mathrm{~g})$.

5. Dado que los pacientes que presentaron efectos secundarios con la dipirona recibieron concomitantemente, en promedio, $7 \pm 2$ medicamentos (rango 3 a 13) y que los pacientes que presentaron efectos secundarios con el tramadol, $7 \pm 3$ medicamentos (rango 2 a 14), se incrementa la probabilidad de experimentar una interacción medicamentosa de cualquier tipo.

6. Llama la atención que el efecto secundario más frecuente para la dipirona fuera la falla terapéutica $(21,59 \%)$. Es de destacar que no se detectaron casos de agranulocitosis; los efectos hematológicos $(15,9 \%)$ detectados correspondieron a 6 casos de leucopenia y a 5 de anemia.

7. Los efectos asociados al tramadol hacen parte del perfil de seguridad establecido para el fármaco, aunque con mayores incidencias a las reportadas. Por ejemplo, el efecto secundario más frecuente fue el estreñimiento $(23,02 \%)$, con una incidencia de 54 por 100.000 pacientes año; el íleo adinámico, la taquicardia y la dificultad respiratoria se presentaron con incidencias de 3,7, 13 y 5,6 por 100.000 pacientes año, respectivamente.

8. El costo total directo por la atención de los efectos secundarios a la dipirona y al tramadol en el hospital, durante el tiempo de estudio (6 meses), fue de $\$ 26^{\prime} 048.281$ o US\$14.346,53. Si se acepta un porcentaje de efectos secundarios prevenibles de $70 \%$, el monto ahorrado para el pagador sería de \$ 18 '233.797 o US\$10.042,57.

9. Vale la pena resaltar que no se encontró ningún caso de agranulocitosis durante la búsqueda en la primera parte de este estudio. Como se había explicado anteriormente, la agranulocitosis es un evento raro cuya incidencia en Europa es de hasta 1 de cada 3.000 pacientes expuestos y, en Latinoamérica, de 1,2 casos de agranulocitosis por un millón de personas 
expuestas a la dipirona hasta por una semana (10). En este caso hay un seguimiento activo con más de 1.390 exposiciones a dipirona sin que se reportara este efecto secundario; por lo anterior, es de notar que, a pesar de las sobredosis utilizadas del medicamento, que seguramente debe alertar a las autoridades sanitarias de la institución para crear planes de control y sensibilización de su uso adecuado.

10.A pesar de las sobredosis utilizadas de dipirona, este medicamento constituye un fármaco eficaz para el tratamiento sintomático del dolor y de la fiebre con un perfil riesgo/ beneficio favorable.

11. Se debe fortalecer la implementación y puesta en marcha de programas institucionales de farmacovigilancia liderados por los comités de farmacia y terapéutica y que, alineados con la actual reglamentación vigente, permitan fortalecer la red nacional de farmacovigilancia e impactar en resultados positivos de uso adecuado de medicamentos (teniendo en cuenta las dosis diarias definidas y plasmándolas en los protocolos de manejo clínico) y prevenir los efectos secundarios en las instituciones prestadoras de servicios de salud. El presente es un estudio piloto que puede ser replicado en otras instituciones de salud.

\section{Conflicto de intereses}

El autor y coautores del presente artículo declaramos que no tenemos conflictos de interés de orden académico, institucional $u$ operacional en el momento de la realización de la investigación.

\section{Financiación}

Esta investigación fue realizada por los autores con el apoyo o tutoría de los profesores de la Universidad Nacional de Colombia, como parte de los requisitos para optar al título de Master en Ciencias-Farmacología. Como tal, la presente investigación contó con financiación por fuente específica del Programa de Investigación del Hospital Universitario de La Samaritana y de la Universidad Nacional de Colombia.

\section{Referencias}

1. Hamerschlak N, Cavalcanti A. Neutropenia, agranulocytosis and dipyrone. Sao Paulo Med J. 2005;123:247-9.

2. Schönhöfer P, Offerhaus L, Herxheimer A. Dipyrone and agranulocytosis: what is the risk? Lancet. 2003; 361:968-9.

3. Plager M. Las preguntas más frecuentes acerca de Novalgina ${ }^{\circledR}$. Sanofi-Aventis. Fecha de consulta: 5 de noviembre de 2006. Disponible en: http://en.sanofiaventis.com/publications/publications.asp

4. Edwards JE, McQuay HJ. Dipyrone and agranulocytosis: what is the risk? Lancet. 2002:360:1438.

5. Sollero L. Incidence of agranulocytosis and the use of dipyrone in Brazil. Rev Bras Pesquisas Med Biol. 1976;9:79-86.

6. Hamerschlak N, Maluf E, Pasquini R, Eluf-Neto J, Moreira FR, Cavalcanti AB, et al. Incidence of aplastic anemia and agranulocytosis in Latin America: the LATIN study. Sao Paulo Med J. 2005;123:101-4.

7. Grond S, Sablotzki A. Clinical pharmacology of tramadol. Clin Pharmacokinet. 2004;43:879-923.

8. Tribiño G, Maldonado C, Segura O, Díaz J. Costos directos y aspectos clínicos de las reacciones adversas a medicamentos en pacientes hospitalizados en el servicio de medicina interna de una institución de tercer nivel de Bogotá. Biomédica. 2006;26:31-41.

9. Grupo de Farmacovigilancia INVIMA/UN. Boletín de Farmacovigilancia. No. 11. Fecha de consulta: 28 de septiembre 28 de 2006. Disponible en http://web. invima.gov.co/Invima//farmacovigilancia/docs_boletines/ BOLETIN\%2011.pdf

10. Segura O, Maldonado C. Las reacciones adversas a medicamentos: una aproximación desde el punto de vista económico. Biomédica. 2003;23:401-7.

11. Hunziker T, Bruppacher R, Kuenzi U, Maibach R, Braunschweig S, Halter $\mathbf{F}$, et al. Classification of ADRs: a proposal for harmonization and differentiation based on the experience of the Comprehensive Hospital Drug Monitoring Bern/St. Gallen, 1974-1993. Pharmacoepidemiol Drug Saf. 2002;11:159-63.

12. Londoño A. PR Vademecum 2008. $7^{a}$ edición. Bogotá: Licitelco S.A.; 2007.

13. Dólar HOY. Cotización del dólar en Colombia. Fecha de consulta: 24 de marzo de 2008. Disponible en: http:// www.wilkinsonpc.com.co/free/dolar-hoy.html.

14. Pucino F, Beck CL, Seifert RL, Strommen GL, Sheldon PA, Silbergleit IL. Pharmacogeriatrics. Pharmacotherapy. 1985;5:314-26

15. Dennis R, Gutiérrez JM, Rodríguez MN. Creación de un programa piloto de farmacovigilancia en el Hospital 
Universitario San Ignacio. Acta Médica Colombiana. 1998;23:15-22.

16. Katzung BG, Bertram G. Farmacología básica y clínica. 10aㅡ edition. East Norwalk: Mc Graw Hill/Appleton \& Lange; 1996.

17. Cadieux RJ. Drug interactions in the elderly. How multiple drug use increases risk exponentially. Postgrad Med. 1989;86:179-86.

18. Schneider JK, Mion LC, Frengley JD. Adverse drug reactions in an elderly outpatient population. Am J Hosp Pharm. 1992;49:90-6.

19. Carty MA, Everitt DE. Basic principles of prescribing for geriatric outpatients. Geriatrics. 1989;44:85-98.

20. Carnevali DL, Patrick M. Nursing management for the elderly. $2^{\mathrm{a}}$ edition. Philadelphia: JB Lippincott Co; 1986.

21. García-Martínez JM, Fresno JA, Lastres P, Bernabéu C, Betés PO, Martín-Pérez J. Effect of metamizol on promyelocytic and terminally differentiated granulocytic cells. Comparative analysis with acetylsalicylic acid and diclofenac. Biochem Pharmacol. 2003;65:209-17.

22. Laporte JR, Capella D. Métodos aplicados en estudios descriptivos de utilización de medicamentos. En: Laporte JR, Tognoni G, editores. Principios de epidemiología del medicamento. $2^{\mathrm{a}}$ edición. Barcelona: Ediciones Técnicas y Científicas S.A; 1993. p. 68-9.

23. Carbonin P, Pahor M, Bernabei R, Sgadari A. Is age an independent risk factor of adverse drug reactions in hospitalized medical patients? J Am Geriatr Soc. 1991;39:1093-9.

24. Nolan L, O'Malley K. Prescribing for the elderly. Part I: Sensitivity of the elderly to adverse drug reactions. J Am Geriatr Soc. 1988;36:142-9.

25. Leape LL, Brennan TA, Laird N, Lawthers AG, Localio AR, Barnes BA, et al. The nature of adverse events in hospitalized patients. Results of the Harvard Medical Practice Study II. N Engl J Med. 1991;324:377-84. 